\title{
An Innovative Approach to Health Education: Utilizing A Functional and Integrative Medicine Education Resource with Students, Clinicians and Patients
}

\author{
Robert D Abbott. ${ }^{1}$ \\ 1. University of Virginia School of Medicine \\ Family Medicine Resident, PGY-1, Virginia Commonwealth University- \\ Shenandoah Valley
}

\section{Contact Information/Degrees/Credentials}

Rob Abbott, MD

448 N. Loudoun St.

Apt B

Winchester, VA 22601

(757) 320-9383

rda4zf@virginia.edu

\section{Introduction:}

According to the World Health Organization a healthcare system involves "all the activities whose primary purpose is to promote, restore or maintain health" (The World Health Report 2000 - Health systems: improving performance). This broad definition encompasses healthcare practitioners, healthcare students and patients. In recent years, the Ancestral Health and Functional Medicine movements have helped empower all three groups. The Institute for Functional Medicine defines Functional Medicine as a medical model that addresses the underlying causes of disease, using a systems-oriented approach, engaging both patient and practitioner in a therapeutic partnership. Ancestral Health can broadly be described as an approach to health focused on optimizing lifestyle habits involving diet, sleep, social interaction, movement and spiritual engagement based on the evolutionary history of our ancestors as well as our more recent cultural relatives. An obstacle for both of these movements, however, is that the delivery of information and educational resources is dispersed over many different organizations and often difficult to access and consume. Books, websites, videos, podcasts and informational handouts can be easily found with the advent of the Internet, but are not easily organized. This extended abstract highlights an electronic book 
resource that organizes Ancestral Health and Functional Medicine resources by learning style (auditory, visual and kinetic) and further categorizes the resources by level of expertise or intended audience. The goal of this educational resource is to provide a free repository of educational health resources accessible to the public that will streamline integrative, ancestral and functional medicine education for practitioners, healthcare students and patients.

\section{Aims and Objectives:}

1. To discuss the availability, utility and accessibility of educational resources with regards to an ancestral, functional and integrative approach to health.

2. To provide a methodical and practical approach for exploring educational resources with regards to identified goals, intentions, and cognitive/learning styles.

3. To allow one to appreciate the need for broadening one's approach and understanding of currently available educational resources including both those that are freely accessible and those available through formal trainings/certification programs.

4. For clinicians, to empower them to expand their education into integrative, ancestral and functional medicine as well as to provide them with a framework to offer concise and individualized educational health resources/plans tailored to the individual interests, concerns and preferred learning style of their patients.

5. For students, to provide them with a practical, complementary educational plan to support their current academic endeavors, tailoring the experience to their available financial resources and preferred cognitive learning style.

6. For patients, to provide them and their families with reliable and relevant educational resources, to empower them to utilize a variety of resources to aid in lifestyle and habit change, and to allow them to appreciate the need for collaboration with an experienced holistic clinician when necessary. 


\section{Methods, Organization and Contents}

Following a reflective introduction, the electronic book organizes a selection of currently available resources across 4 categories:

1. Video Resources

2. Text Resources (Blogs, E-Book, Books)

3. Audio Presentations (Podcasts)

4. Conferences and Trainings

Resources were selected based on the reputability of the information and the background of the researcher, clinician, or health advocate providing the information. Resources were also selected based on relevance, accessibility and scientific veracity. Efforts were also made to include resources from a diverse background of researchers, clinicians, and health advocates, reflecting the diverse population consuming the educational material.

Example Entries From Each Section

\section{A. Video Resources}

\section{The Evolution of Medicine's Functional Forum}

The Functional Forum is the world's largest integrative medicine conference. On the first Monday of every month, this new concept in practitioner education, "medutainment", brings together the latest health news, functional medicine research, practice development and health technology in an upbeat, entertaining way.

Tip for Getting Started: Start with The Evolution of Medicine's Functional Forum. This will be by far your greatest value in terms of time and clinical relevance. The episodes are jam packed with panels, talks and group discussions.

https://www.youtube.com/user/functionalforum 


\section{B. Text Resources}

\section{Stephan Guyenet PhD: Whole Health Source: Nutrition \& Health Science}

Stephan is a longtime contributor within the world of Ancestral Health. Stephan's blog contains a vast collection of accessible articles synthesizing the latest research on all things nutrition, obesity, and insulin resistance. A great place for patients who want concise and readable analyses of the scientific literature and for scientists or health practitioners who want objective, unbiased and reliable interpretations of newly published literature.

Blog: A nice variety of scientific inquiry with reflective personal commentary.

\section{http://wholehealthsource.blogspot.com}

Note: Stephan has recently moved his content to a new page following the release of his new book: The Hungry Brain: Outsmarting The Instincts That Make Us Overeat

Check out the new page here: http://www.stephanguyenet.com

\section{Auditory Resources (Podcasts)}

\section{Chris Kresser's Revolutionary Health Radio}

Functional, ancestral medicine at its core. Start here and you won't be disappointed or wanting for thoughtful episodes and engaging interviews. Accessible for both the engaged patient and curious health practitioner.

\section{https://chriskresser.com/podcasts/}

\section{Dr. Ruscio Radio}

A great variety of interviews and practical clinical content. Weekly down to earth episodes that will broaden your knowledge of cost-effective functional medicine.

\section{https://drruscio.com/podcasts/}




\section{Chris Masterjohn PhD: Mastering Nutrition}

If you thought you knew nutritional biochemistry, think again. For those of you who like two hour rabbit hole discussions on glutathione, this is a wonderful place to start. In all seriousness, follow Chris' work. He is an incredible teacher.

\section{https://chrismasterjohnphd.com/podcast/}

\section{Conferences and Trainings}

\section{Ornish Lifestyle Medicine:}

Combining the incredible healing powers of stress management, nourishing nutrition, fitness and movement with the elixir that is love and connection, Dr. Dean Ornish has recently partnered with Healthways in order to train more multidisciplinary teams in his unique lifestyle approach. You will need your friends and colleagues for this one, as it does involve multiple team members, but this program, indeed, has the power to change the lives of thousands.

\section{https://www.ornish.com/site-certification/\#section training-certification}

\section{Conclusions}

Health education and its delivery are changing. Medical school costs are exceeding \$200,000 and the demand for integrative and functional clinicians well versed in lifestyle and root-cause approaches to health is outpacing the supply. Current students are often unaware of ancestral and functional approaches to wellness and are easily overwhelmed by the breadth of information provided as part of their formal education. Patients and laypersons too, are not immune to the growing problem of information overload, as the coming generations will not be faced with the problem of going without, but rather, with the problem of having too much. Collectively, practitioners need educated and empowered patients and patients need educated and open-minded clinicians. We are a worldwide community needing change in order to promote health and need each other to thrive. This dynamically evolving electronic book is a first attempt at consolidating some of the most relevant health educational resources, so students, clinicians and patients do not have to search in vain for what can bring them joy and health. 


\section{References}

(See the contents of the electronic book for all of the direct links to resources) 